\title{
Influence of single dye molecules on temperature and time dependent optical properties of CdSe/ZnS quantum dots: Ensemble and single nanoassembly detection
}

\author{
Eduard I. Zenkevich $^{\mathrm{a}, *}$, Alexander P. Stupak ${ }^{\mathrm{b}}$, Danny Kowerko ${ }^{\mathrm{c}, 1}$, Christian von Borczyskowski $^{\mathrm{c}}$ \\ ${ }^{a}$ National Technical University of Belarus, Department of Information Technologies and Robotics, Nezavisimosti Ave., 65, Minsk 220013, Belarus \\ ${ }^{\mathrm{b}}$ B.I. Stepanov Institute of Physics, National Academy of Science of Belarus, Nezavisimosti Ave., 70, 220072 Minsk, Belarus \\ ${ }^{c}$ Institute of Physics and Center for Nanostructured Materials and Analytics (nanoMA), Chemnitz University of Technology, 09107 Chemnitz, Germany
}

\section{A R T I C L E I N F O}

Article history:

Available online 20 February 2012

\section{Keywords:}

Semiconductor quantum dots

Porphyrins

Self-assembly

Ligand dynamics

Surface traps

Fluorescence resonant energy transfer

(FRET)

Non-FRET photoluminescence quenching

Temperature surface "phase" transition

\begin{abstract}
A B S T R A C T
Optical spectroscopy on ensembles and single CdSe/ZnS semiconductor quantum dots (QDs) demonstrates a competition of trap and near band edge photoluminescence (PL). This competition can be markedly influenced by a few surface attached pyridyl functionalized dye molecules (porphyrins or perylene diimides) forming nanoassemblies with well defined geometries. Temperature variation and related changes in absorption and emission reveal sharp changes of the ligand shell structure in a narrow temperature range for organic (TOPO and amine) surfactants (phase transition). The effects on QD PL at this transition become considerably pronounced upon attachment of only a few dye molecules to QD surface. Moreover, under ambient conditions amine capped QDs are photodegraded in the course of time. This process is enhanced by attached dye molecules both on the ensemble and single particle/dye level. This investigation elaborates the importance of (switchable) surface states for the characterization of the PL of QDs.
\end{abstract}

(c) 2012 Elsevier B.V. All rights reserved.

\section{Introduction}

Recently, great efforts have been devoted to bottom-up nanostructures. This results from their versatile and controllable geometric features with closely related structure-dependent properties [1-5]. Much attention has been paid to the investigation of physicochemical and optical properties of nanostructures based on colloidal semiconductor quantum dots (QDs) $[6,7]$ being used in biomedicine $[8,9]$ or as components of optoelectronic nanodevices [10], chemical or biochemical nanosensors, energy or information storage, and nanocatalysis $[11,12]$.

Of special interest is the formation of QD-dye nanoassemblies, since they allow for a controlled realization of mutually relative (spatial) orientations and electronic energy scales in order to optimize intended photoinduced processes such as charge transfer [13-15], fluorescence resonance (Foerster) energy transfer (FRET) [16-21] or electron tunneling in the conditions of quantum confinement $[22,23]$. Basically there are several routes to realize nanoassemblies in liquid solutions and polymeric matrices in the form of (i) blends [24], (ii) QD-polymer-dye composites [16,25], and

\footnotetext{
* Corresponding author. Tel.: +37517293 9123; fax: +37517297153.

E-mail address: zenkev@tut.by (E.I. Zenkevich).

1 Present address: University of Zürich, Institute for Inorganic Chemistry, 8057 Zūrich, Switzerland.
}

(iii) via functional groups self-assembled QD-dye aggregates $[14,17,19,20,22,26]$.

With respect to formation of self-assembled QD-aggregates in the liquid phase several factors related to the QD photoluminescence (PL) efficiency are of essential importance: (i) attachment/ detachment of dye molecules [14,16,17,19,22,26], (ii) the interplay of dye molecule attachment and ligand exchange dynamics $[23,27,28]$, and (iii) the presence and formation of various surface trap states in the band gap [29,30], whose energies and corresponding PL quenching efficiencies may be changed upon (temperature dependent) interface reconstruction or competing ligand/dye exchange dynamics. In the past a few studies have been devoted to the elucidation of thiol-type ligand exchange at relative low concentrations between 1 and 10 relative to the QD concentration $[28,31]$. In this respect, the combination of bulk and single molecule/single particle experiments [32] are a tool to precisely identify the interaction of exactly one QD with one dye molecule leading to a microscopic understanding of the formation (including ligand dynamics) and related mechanisms of PL quenching dynamics for QD-dye nanoassemblies.

Quantum efficiency and PL dynamics for QDs depend drastically on dielectric properties of the embedding environment [33] and temperature [51,34-37]. Very recently, we have shown [23] that at ambient temperature in liquid solutions, the formation of QD-dye nanoassemblies (based on CdSe QDs and meso-pyridyl 
porphyrins [19] or perylene diimides) [32]) takes place in competition with capping ligand (surfactant) dynamics and is realized at least on two time scales which is attributed to a reorganisation of the capping ligand shell. We established a microscopic description of PL quenching and assembly formation in the conditions of extremely low concentrations of QDs and dye molecules [23].

As a part of our detailed analysis of self-assembly processes in QD-dye nanoassemblies, we report in this paper both on the influence of structural surface transformations such as a ligand induced phase transition and photodegradation on the optical properties of QDs, especially how they are related to assembly formation. Our comparative results are concerned with (77-300 K) temperature dependent optical properties of QD-porphyrin nanoassemblies (ensemble experiments) as well as on long-term temporal evolution of PL spectra (photodegradation related to interface transformation processes) of QDs assembled with perylene diimide (ensemble and single particle detection). We have chosen porphyrin-type dye molecules, since due to their versatile functionalisation well defined $\mathrm{QD}$-porphyrin nanoassembly structures can be realized $[19,20,22]$. However, porphyrins are not suitable candidates for single molecule detection due to the relatively low fluorescence quantum efficiency. Perylene diimide-type dyes are, on the other hand, one of the most ideal single probe molecule accompanied with a high photostability. In case of porphyrin we will report on the (temperature dependent) formation of $\mathrm{CdSe} / \mathrm{ZnS}$ trap states in competition to QD near band edge states, while the temporal variation of trap and near band edge states will be investigated with a diimide dye.

\section{Experimental}

\subsection{CdSe quantum dots}

For ensemble and single particle experiments colloidal core/ shell CdSe/ZnS QDs passivated by trioctylphosphine oxide (TOPO) or long-chain amines (AM) were obtained from Evident Technologies, Inc., Troy, NY, USA. The absorbance of the QD starting toluene solution was adjusted to be lower than 0.1 OD at excitation and emission wavelengths in order to avoid non-linear absorption and re-absorption effects. The concentrations varied in the range $(1-10) \times 10^{-7} \mathrm{M}$. In ensemble experiments stability and purity of the QD solutions were checked by measuring the quantum yield stability at least over $3 \mathrm{~h}$ after preparation.

\subsection{Functionalized dye molecules}

5,10,15,20-meso-metapyridyl substituted porphyrin (m$\mathrm{Pyr})_{4} \mathrm{H}_{2} \mathrm{P}$ and its Cu-derivative, (m-Pyr $)_{4} \mathrm{CuP}$ were synthesized and purified according to known methods described by us previously [38]. It is known from our recent publications [19,20,39] that among a series of pyridyl-substituted free-base porphyrins and chlorins, tetra-meso-metapyridyl substituted porphyrins are characterized by an extremely large complexation constant upon formation of QD-porphyrin nanoassemblies. In ensemble experiments, the porphyrin stock solution was prepared in toluene under ultrasonic treatment at $40^{\circ} \mathrm{C}$ at concentrations in the range $(3-30) \times 10^{-5} \mathrm{M}$ and then was used for the formation of "QDporphyrin" nanoassemblies via titration of QD solutions. For single particle experiments, sufficiently high photostable pyridyl functionalized perylene diimide molecules were used. The synthesis of these compounds is described elsewhere [40,41]. In the present experiments we have used (pyridyl) ${ }_{1}$-perylene diimide (PP). The dye molecules and QD-dye assemblies are shown in Fig. 1.

\subsection{Sample preparation}

Quantitative titration experiments have been performed in case of ensembles at ambient conditions with toluene spectroscopic grade (Fluka SeccoSolv or Merck dried with a molecular sieve). The optical cuvettes (Hellma QS-111, path length $1 \mathrm{~cm}$ ) and other glassware were flushed with acetone and ethanol, chemically cleaned with aqueous $\mathrm{H}_{2} \mathrm{SO}_{4}: \mathrm{H}_{2} \mathrm{O}_{2}$, flushed with deionized water, dried in a nitrogen flow and purged with toluene. Temperature (77-300 K) measurements for QDs and "QD-porphyrin" nanoassemblies were carried out in a methylcyclohexane/toluene (6:1) mixture possessing an optical transparent glass at low temperatures. The respective glass transition temperatures are the following: $146.7 \mathrm{~K}$ for pure methylcyclohexane, $180 \mathrm{~K}$ for pure toluene [42], and $151.6 \mathrm{~K}$ for methylcyclohexane/toluene (6:1) mixtures [43]. For single particle experiments samples, pipettes and vials were thoroughly cleaned by supersonic treatment in a piranha solution (1:1 $\mathrm{H}_{2} \mathrm{O}_{2}-\mathrm{H}_{2} \mathrm{SO}_{4}$ solution) and by careful rinsing with Millipore DI water. Material quality was checked with a homebuilt fluorescence wide field microscope. Single particle sample preparation was done by spin coating a $5 \times 10^{-11} \mathrm{M}$ solution of the QD-dye mixture onto a $\mathrm{Si} / \mathrm{SiO}_{2}\left(100 \mathrm{~nm}\right.$ thick $\left.\mathrm{SiO}_{2}\right)$ or quartz surface. Structures of 5,10,15,20-meso-metapyridyl substituted $\mathrm{Cu}$ porphyrin (CuP) and (pyridyl) 1 $_{1}$-perylene diimide (PP) molecules, capping molecules of trioctylphosphine oxide (TOPO) and longchain amines (AM) are depicted in Fig. 1. This figure shows also a schematic representation (not on scale) of "QD-porphyrin" (A) and "QD-perylene diimide" (B) nanoassemblies and mutual arrangement of dye molecules with respect to QD surface.

\subsection{Experimental techniques}

In ensemble experiments, absorption spectra were recorded with a Shimadzu $3001 \mathrm{UV} /$ Vis or Cary-500 M Varian spectrometer. Emission spectra were measured with a SFL-1211A (Solar, Belarus) or Shimadzu RF-5001PC spectrofluorimeter. Temperature experiments were carried out using a home built cryostat (Solar, Belarus) equipped with a temperature controller $(\Delta T=0.5 \mathrm{~K})$. Single particle experiments at ambient conditions were performed in a home built laser scanning confocal microscope described earlier [32].

\section{Results and discussion}

\subsection{Surfactant related phase transitions in $Q D-d y e$ nanoassemblies}

We have carried out detailed QD absorption spectra (in the energy range of the first excitonic transition), photoluminescence (PL) spectra, and PL excitation (PLE) spectra at various detection wavelengths as well as PL decays at various temperatures and excitations or detection conditions. Experiments on ensembles of CdSe/ $\mathrm{ZnS}$ QDs ( $d_{\mathrm{CdSe}}=3.0 \mathrm{~nm}, 2 \mathrm{ZnS}$ capping monolayers) have been performed in a methylcyclohexane/toluene (6:1) mixture in a temperature range from $77 \mathrm{~K}$ to $300 \mathrm{~K}$. A set of typical $\mathrm{CdSe} / \mathrm{ZnS}$ spectra is depicted in Fig. 2 for various temperatures. While the detailed results are still under investigation and will be discussed in a forthcoming paper, we will concentrate in this publication on the temperature dependent influence of dye molecules on the QD PL and will only present those results on ensembles of QDs, which are necessary to follow the overall behavior.

Fig. 2A shows that the band-edge absorption for CdSe/ZnS QD shifts to the blue upon temperature lowering. It was found that the optical density remains nearly constant at various temperatures for wavelengths below $450 \mathrm{~nm}$ (accuracy $\pm 2.5 \%$ ). At $\lambda=520 \mathrm{~nm}$ it varies less than $\pm 25 \%$. Temperature dependent QD PL spectra are presented in Fig. 2B for excitations at $450 \mathrm{~nm}$ and 


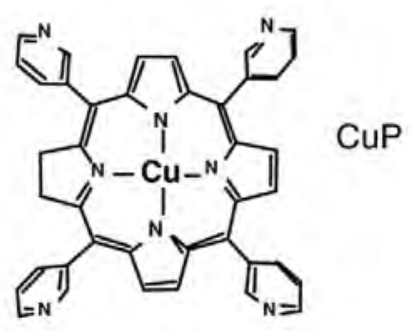

A
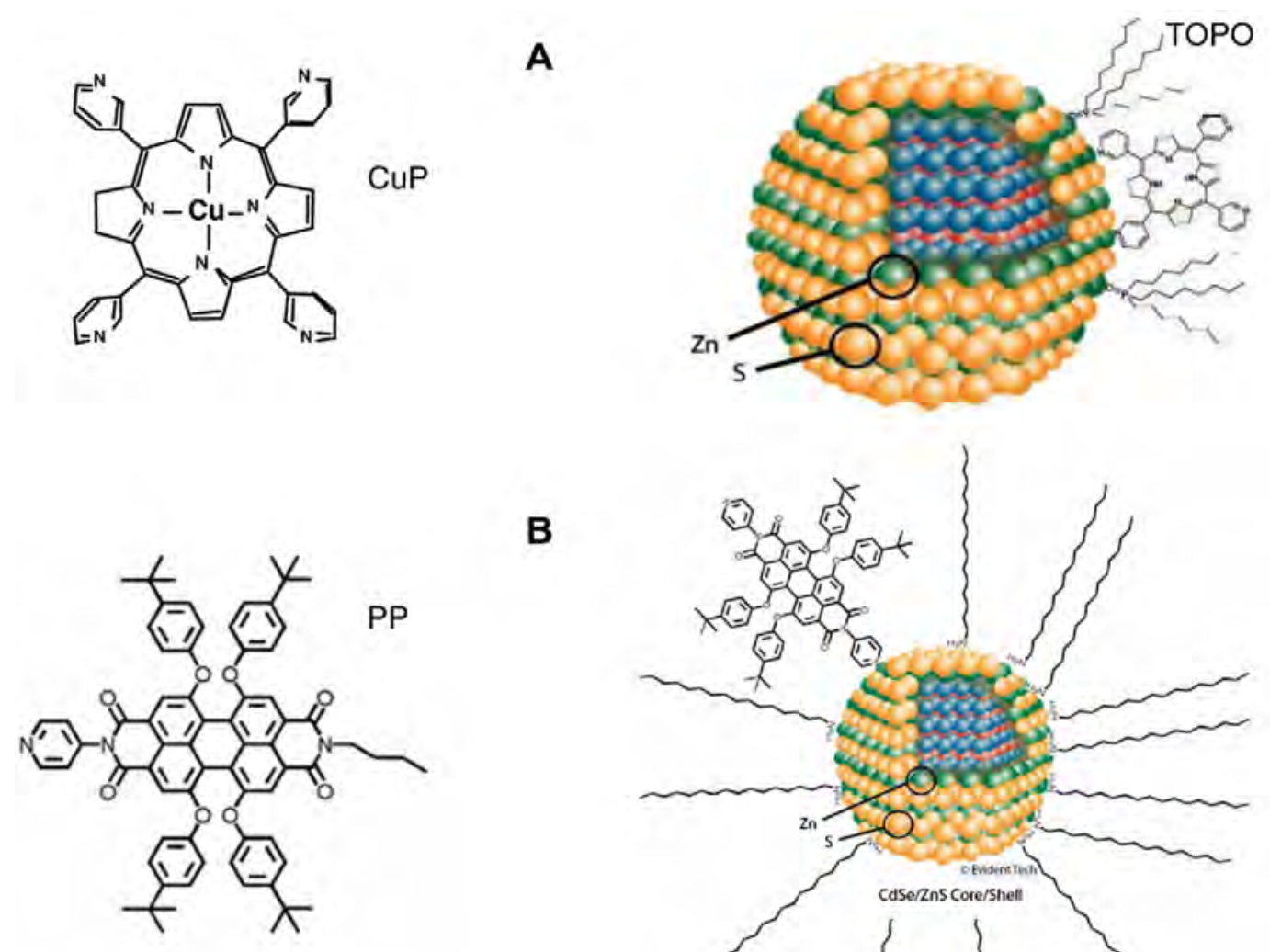

B

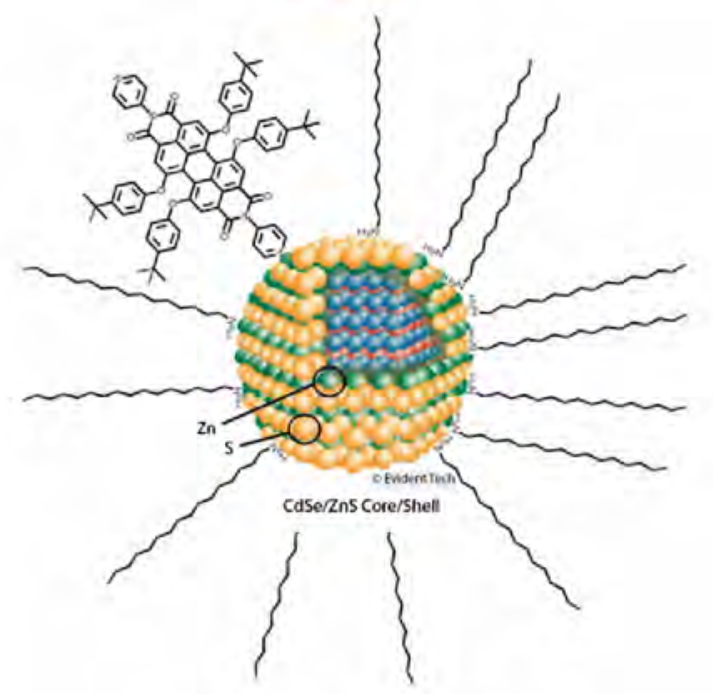

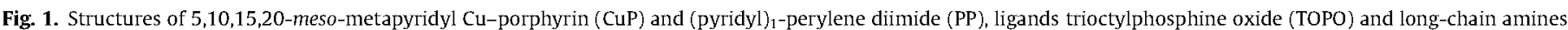

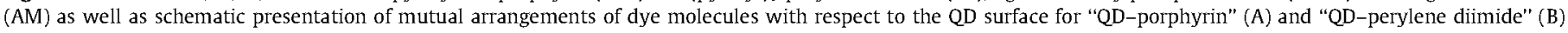
nanoassemblies.

A

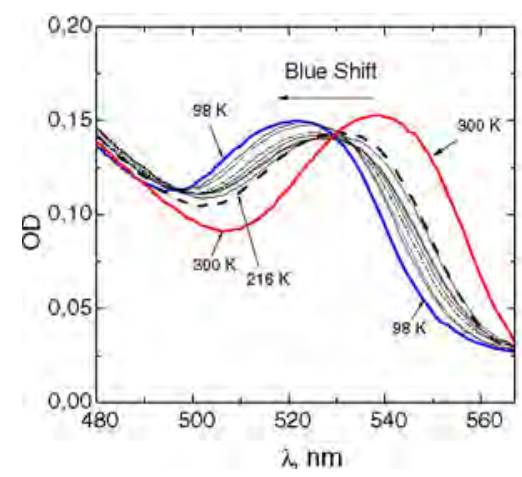

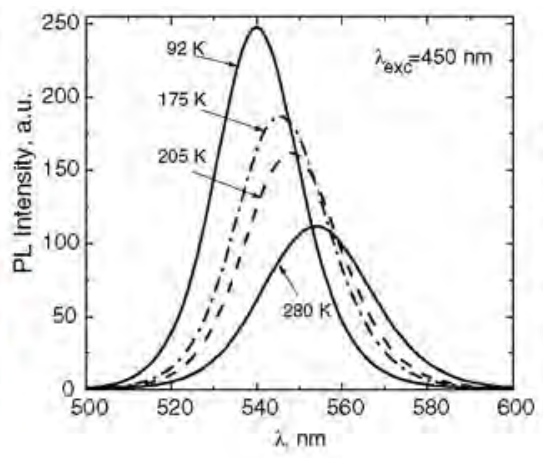

B

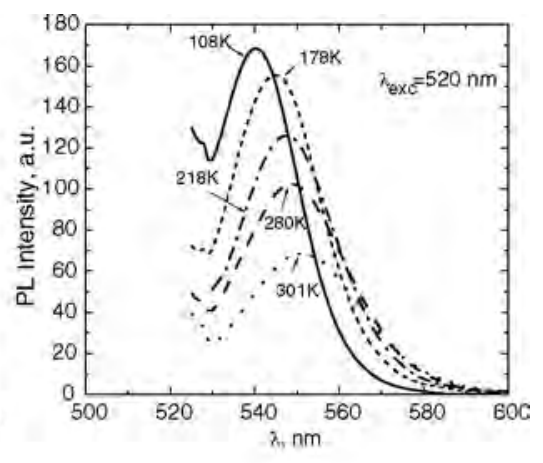

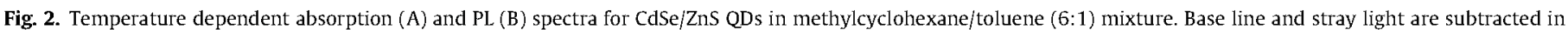

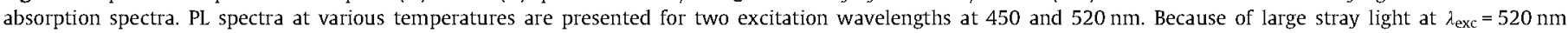
$\left(\lambda_{\text {exc }}=5 \mathrm{~nm}\right)$ the related PL spectra are depicted beginning at $\lambda_{\mathrm{PL}}=530 \mathrm{~nm}$.

$520 \mathrm{~nm}$, respectively. The temperature dependence of the PL peak energy behaves similar as the band-edge absorption, that is it is shifted to the blue upon temperature lowering. At $295 \mathrm{~K}$ excitation between 410 to $500 \mathrm{~nm}$ results in a PL band at $\lambda_{\max }=555 \mathrm{~nm}$, while upon excitation in the range of $510-540 \mathrm{~nm}$ the PL band is characterized by $\lambda_{\max }=551 \mathrm{~nm}$. We conclude that in addition to site selection (observed for QD PL [44]) our data reveal the presence of at least two luminescent states. At $T<108 \mathrm{~K}$ the PL dependence on $\lambda_{\text {exc }}$ becomes essentially weaker. Upon temperature lowering PL spectra are not only shifted to the blue, but also the line width (FWHM) becomes essentially narrower, while the PL intensity becomes higher. Taking into account the nearly constant optical density (see above), this implies that the PL quantum efficiency increases upon temperature lowering and PL emission takes place presumably from only one type of state.

Fitting the lowest absorption and PL band position in the energy scale as a function of temperature by a Gaussian line, we observe besides the already mentioned blue shift upon temperature 


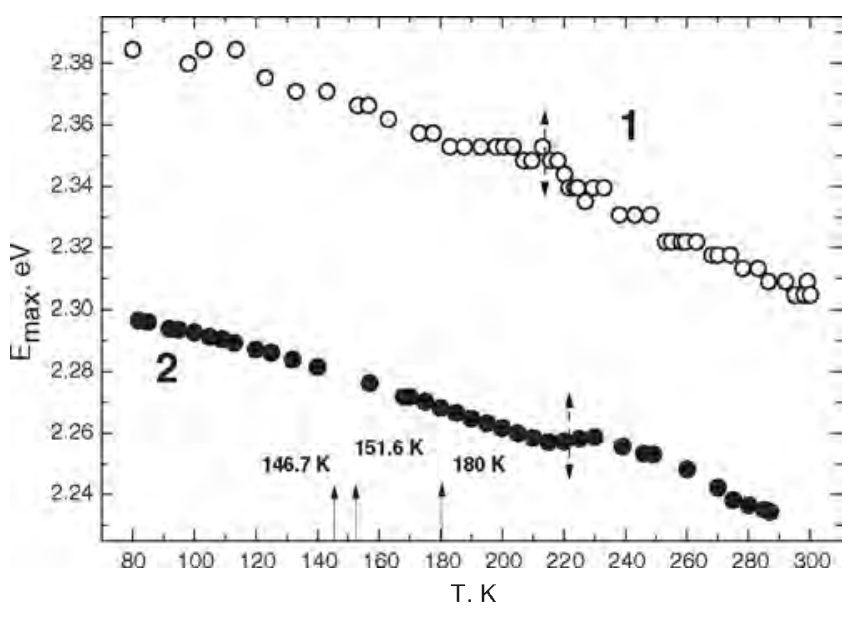

Fig. 3. Comparison of temperature dependence of (1) the first absorption peak (mean weighted data for two Gaussians lines) and (2) PL maximum $\left(\Delta \lambda_{\mathrm{exc}}=450 \mathrm{~nm}\right)$ for TOPO capped CdSe/ZnS QDs in methylcyclohexane/toluene $(6: 1)$ mixture. Thin arrows in the bottom indicate glass transition temperatures for methylcyclohexane $(146.7 \mathrm{~K})$, methylcyclohexane/toluene $(6: 1)$ mixture $(151.6 \mathrm{~K})$ and toluene $(180 \mathrm{~K})$, respectively. Thick dashed arrows on the curves 1 and 2 indicate temperatures at which optical properties change.

lowering a non-monotonous temperature dependence of the respective peak energies. As can be seen in Fig. 3 this non-monotonous behavior (which we name for simplicity "kink") is observed for TOPO-capped CdSe/ZnS QDs between 200 and $240 \mathrm{~K}$, far from the glass transition temperatures for methylcyclohexane $(146.7 \mathrm{~K})$ and toluene $(180 \mathrm{~K})$, respectively [42], as well as for a methylcyclohexane/toluene (6:1) mixture (151.6 K) [43]. Fig. 3 shows that with lowering temperature the "kink" occurs at slightly different temperatures for the first excitonic absorption band $(\sim 218 \mathrm{~K})$ and the PL band $(\sim 223 \mathrm{~K})$, respectively. It follows that the observed non-monotonous temperature behavior is reversible both in absorption and PL measurements upon cooling or heating QD samples without any hysteresis effects (see Supplementary materials). In addition, we also found, that a similar "kink" is observed in the PL temperature dependence for CdSe/ZnS QDs capped by long-chain amines (see Fig. 4). In the later case, the "kink" in PL

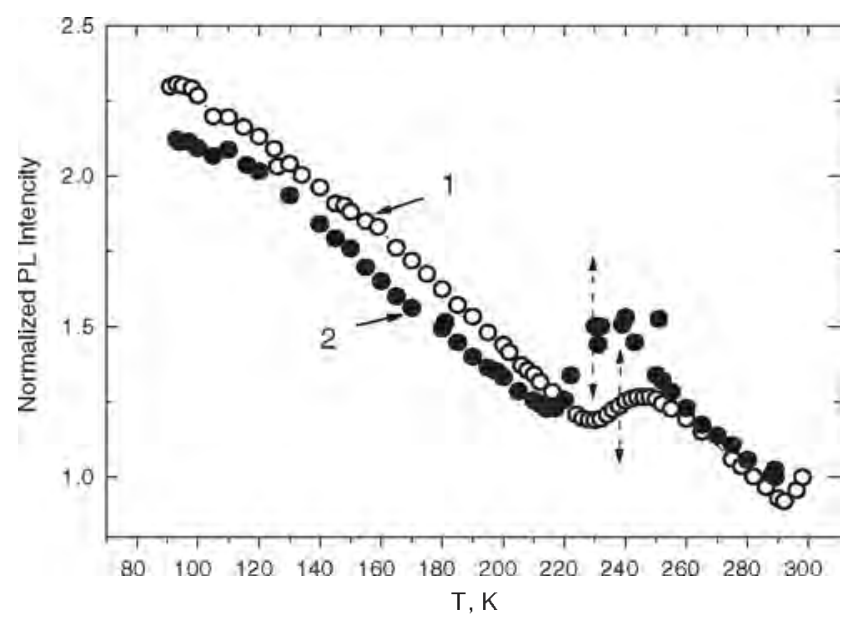

Fig. 4. Temperature dependence of the normalized PL maximum intensity $\left(\lambda_{\text {exc }}=450 \mathrm{~nm}\right)$ for CdSe $/ \mathrm{ZnS}-\mathrm{AM} \mathrm{QDs}\left(d_{\mathrm{CdSe}}=3.0 \mathrm{~nm}, 2 \mathrm{ZnS}\right.$ monolayers $)$ capped by long-chain amines (1) and for nanoassemblies based on CdSe/ZnS-AM QDs and (pyridyl) 2 $_{2}$-perylene diimide (DPP) dye molecules at molar ratio $x=\left[C_{\mathrm{DPP}}\right] /\left[C_{\mathrm{QD}}\right]=1$ (2) in a methylcyclohexane/toluene (6:1) mixture. Thick dashed arrows on the curves 1 and 2 indicate temperatures at which optical properties change. spectra is more pronounced and shifted to $\sim 237 \mathrm{~K}$ as compared to $\sim 223 \mathrm{~K}$ for TOPO-capped CdSe/ZnS QDs. Notably, this non-monotonous behavior becomes stronger for amine -capped CdSe/ZnS QDs with attached DPP dye molecules at molar ratio $x=\left[C_{\mathrm{DPP}}\right] /$ $\left[C_{\mathrm{QD}}\right]=1$ (Fig. 4, curve 2 ), while no "kink" is observed when following the luminescence of perylene diimide or porphyrin molecules under the same conditions (will be presented and discussed below). From these observations, we therefore exclude, that the morphology of the matrix is changing.

To understand the appearance of the "kink" both in absorption and PL temperature experiments on CdSe/ZnS QDs, we refer to observations recently reported in literature. Typically, when increasing temperature quenching of QD PL intensity is a commonly observed phenomenon, both in colloidal suspensions or in solvent-free systems (polymeric matrices or QD solids). Generally this finding is assigned to thermally activated carrier trapping $[17,45]$ or increased electron phonon coupling [31,36,46-50]. Unusual "luminescence anti-quenching" at a well defined temperature of $T \sim 250 \mathrm{~K}$ (manifested in our experiments as "kink") has been observed earlier [36,51] for CdTe or CdSe/ZnS QDs initially dissolved in liquid solutions such as toluene. This effect has been related to a phase transition in the surfactant (ligand) layer [36,51]. As was shown for colloidal (TOPO-capped) CdSe QDs in anhydrous toluene [36] and for water-soluble aminoethanethiol- (AEP-) capped CdTe QDs [51], a phase transition of the surfactant layer leads to surface relaxation and/or surface reconstruction which is strongly dependent on the type of ligand and, in turn, may cause a spatially-energetic reordering of trap states.

The above described effects have up to now only been reported with respect to the PL properties of QDs. In our case, in addition to $\mathrm{PL}$, the QD band-edge absorption is obviously also sensitive to such a phase transition of the surfactant layer at temperatures close to $\sim 218 \mathrm{~K}$. Now let us turn in more detail to the temperature dependence of the QD band-edge absorption (Fig. 3). In the high temperature range $(T>250 \mathrm{~K})$ it has been experimentally shown (and theoretically confirmed) that experimental values of $d E / d T$ are independent of the size of CdSe/ZnS QDs and they agree with the average bulk value of $d E_{\text {bulk }} / d T=-0.36 \mathrm{meV} / \mathrm{K}$ [48]. The results depicted in Fig. 3 give $d E / d T=-0.42 \mathrm{meV} / \mathrm{K}$ below and $d E /$ $d T=-0.32 \mathrm{meV} / \mathrm{K}$ above the phase transition temperature for the band edge absorption. This coincides with data obtained for uncapped CdSe in a poly-laural methacrylate matrix [48] though in the later case, the experimental temperature dependence of the band gap $E(T)$ did not show any "kink".

In addition, when discussing the appearance of the "kink", one has to take into account that for TOPO- or amine-capped CdSe/ZnS QDs (dissolved in various solvents), a phase transition of the ligand layer will cause some strain-induced deformation of the ZnS shell. According to [52-54] absorption and PL of core-shell QDs such as $\mathrm{CdSe} / \mathrm{ZnS}$ are red-shifted with respect to merely core QDs because in the former case the electron wave function tunnels into the shell, increasing the delocalization of the electron-hole pair, lowering the confinement energy and consequently the energy of the excited state. Correspondingly, due to different thermal expansion coefficients for the semi-rigid surfactant layer and the $\mathrm{ZnS}$ shell as well as possible strain effects, slight changes of electron delocalization may take place in the range of the phase transition, that show up in slight differences in $d E / d T$ below and above the phase transition.

Now let us turn to details of the temperature dependence of the PL. Though qualitatively very similar to the absorption, it is obvious from Fig. 3, that $d E / d T=-0.30 \mathrm{meV} / \mathrm{K}$ above the phase transition is very similar to the one of the absorption, but definitely smaller in comparison with the value $d E / d T=-0.43 \mathrm{meV} / \mathrm{K}$ below the phase transition. From this we conclude that, PL at low temperatures does not originate from those (band-edge) states which give 
rise to the absorption. In this respect, we believe that slightly different "kink" temperatures for the first excitonic absorption band $(\sim 218 \mathrm{~K})$ and the PL band ( $\sim 223 \mathrm{~K}$ ) for TOPO-capped CdSe/ZnS QDs, may be explained by the different nature of the corresponding transitions in absorption and in emission. In its turn, the more pronounced and shifted to $237 \mathrm{~K}$ "kink" in the PL spectra for aminecapped CdSe/ZnS QDs (Fig. 4) relative to TOPO-capped CdSe/ZnS QDs (Fig. 3) may be connected with different surface relaxation/ reconstruction which is strongly dependent on the ligand type [36,51].

Additional information is obtained, when inspecting the PL spectra as a function of temperature, which is shown Fig. 5A. At the same temperature range where the "kink" shows up, both in absorption and PL energy, a decrease of the PL intensity of about $5 \%$ (see Fig. 5B, curve 1) is observed, keeping in mind that also the nature of emitting states has changed from a near band-edge emission to a trap dominated emission. If in fact, a trap state emission dominates at low temperatures, these trap states or their distribution might be influenced by dye molecules attached to the QD surface due to replacing some of the surfactant (TOPO ligand)
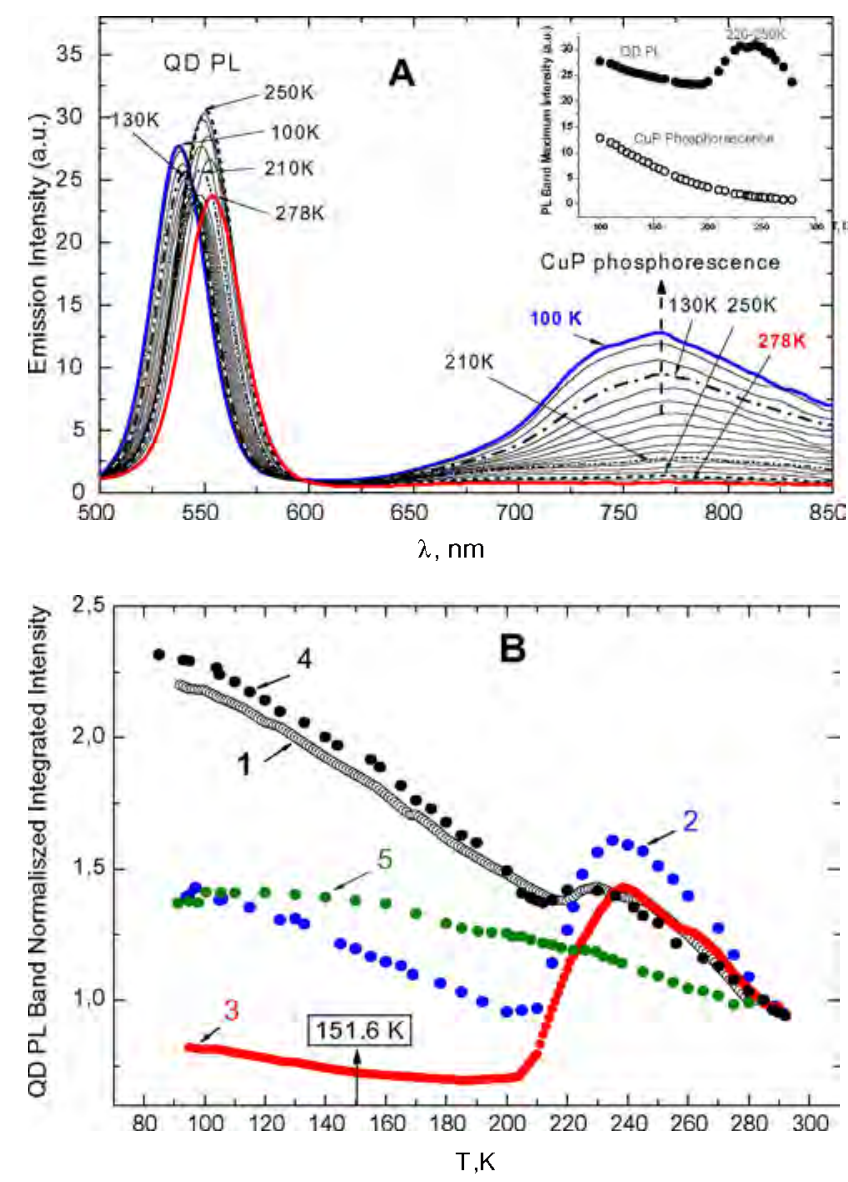

Fig. 5. Temperature dependence of emission spectra (A) and band maxima intensities (B) for nanoassemblies based on TOPO-capped CdSe/ZnS QDs and various porphyrin molecules at molar ratio $x=\left[C_{\mathrm{Porphyin}}\right] /\left[\mathrm{C}_{\mathrm{OD}}\right]=1$ in methylcyclohexane/toluene $(6: 1)$ mixture $\left(\lambda_{\mathrm{exc}}=450 \mathrm{~nm}\right)$. A: The short wavelength band belongs to $\mathrm{QD} \mathrm{PL}$, the increasing long wavelength band belongs to (m-Pyr) ${ }_{4} \mathrm{CuP}$ phosphorescence. The inset shows the temperature dependence for QD PL and CuP phosphorescence bands between 77 and $285 \mathrm{~K}$. B: All curves are normalized to the integrated band intensity of QD PL at $285 \mathrm{~K}$. Experimental temperature dependences are shown for the same type of $\mathrm{QD}\left(d_{\mathrm{CdSe}}=3.0 \mathrm{~nm}, 2 \mathrm{ZnS}\right.$ monolayers $)$ in under various conditions: (1) CdSe/ZnS QDs; (2) QD + (m-Pyr $)_{4} \mathrm{H}_{2} \mathrm{P}$; (3) QD + (m$\mathrm{Pyr})_{4} \mathrm{CuP}$; (4) QD + tetraphenylporphyrin (having no pyridyl rings); (5) QD in dry PMMA film on a quartz plate. The phase transition temperature of the matrix is indicated. molecules $[23,27,28]$. To realize such a situation we titrated a solution of QD by various porphyrin molecules up to a relative molar ratio of $x=\left[C_{\text {Porphyrin }}\right] /\left[C_{\mathrm{QD}}\right]=1$. As has been recently demonstrated $[19,20,39]$ selective pyridyl-functionalization (see Fig. 1) allows for well defined attachments to the QD surface and results in a nanoassembly formation. In Fig. 5A we show for the relative concentration ratio $x=1$ a series of luminescence spectra of $\mathrm{CdSe} / \mathrm{ZnS}-\mathrm{CuP}$ nanoassemblies at various temperatures. The long wavelength emission band at $\lambda_{\max }=770-780 \mathrm{~nm}$ appearing upon temperature lowering belongs to the phosphorescence of (m-Pyr) ${ }_{4} \mathrm{CuP}$ molecules [38] and references therein]. According to [55] it takes place from the ${ }^{2} \mathrm{~T}_{1}$ state (at room temperature) or the ${ }^{4} \mathrm{~T}_{1}$ state (at low temperatures) being caused by exchange $d-\pi$ interactions of the $\mathrm{Cu}$ unpaired d-electron with $\pi$-electrons of the porphyrin ring. Fig. $5 \mathrm{~A}$ (inset) compares the temperature dependence of peak emission intensities for $\mathrm{QD}$ and (m-Pyr) ${ }_{4} \mathrm{CuP}$ molecules after CdSe/ZnS-CuP nanoassemblies have formed. It is evident that the $\mathrm{CuP}$ phosphorescence intensity is continuously increasing upon temperature lowering. A similar monotonic increase of (m$\mathrm{Pyr})_{4} \mathrm{H}_{2} \mathrm{P}$ fluorescence is observed for CdSe/ZnS QD $+(\mathrm{m}-\mathrm{Pyr})_{4} \mathrm{H}_{2} \mathrm{P}$ (1:1) solution upon temperature lowering (not shown). These monotonic temperature dependences of emission are typical for $\mathrm{Cu}$-containing porphyrins and porphyrin free bases in solid solutions and polymers $[38,55]$. In contrast, for $\mathrm{CdSe} / \mathrm{ZnS}-\mathrm{CuP}$ nanoassemblies, the CdSe/ZnS QD PL intensity shows again a "kink", which, however, is now much more pronounced $(-23 \%)$ than in the absence of CuP (compare curves 1 and 3, Fig. 5B). Similar experiments have been performed with other dye molecules, including tetraphenylporphyrin without pyridyl groups, which, however, does not enlarge the PL decrease at the "kink" (curve 4, Fig. 5B). A comparison of temperature dependent CdSe/ZnS PL intensities obtained for various porphyrins is shown in Fig. 5B. It is evident, that dye attachment enhances the PL decrease at the phase transition temperature. The amplification is strongest for CuP and amounts to about $-50 \%$ on the scale of integrated QD $\mathrm{PL}$ intensities. We suggest that $\mathrm{CuP}$ exhibits the largest effect, since either internal molecular charge transfer states influence the energy distribution of CdSe/ZnS trap states considerably or TOPO ligands may be strongly coordinated at low temperatures to the central $\mathrm{Cu}$ ion, which might increase the disorder of the surfactant shell. Moreover, $d E / d T$ below the phase transition is considerably lower in case of assembly formation than that for CdSe/ZnS without dye attachment, which again is a manifestation of newly generated trap states. Finally, it is seen from Fig. 5B that for QDs in a dry PMMA film on a quartz plate that the "kink" is absent (curve 5).

When defining the temperature at the mid-intensity of the "kink" (see e.g. Figs. 3-5) as the apparent phase transition temperature, this temperature is about $5 \mathrm{~K}$ higher for QDs as compared to QD-dye nanoassemblies. Also in case of amine ligands we observe a similar phase transition behavior as is shown in Fig. 4. Again, attachment of dye molecules enhances the decrease of PL intensity and shifts the phase transition to lower temperatures but to a much less extent than observed for TOPO ligands. The noticeable dependence on the type of ligands is in agreement with earlier observations [36,51] and supports the suggestion that a phase transition of ligands couples to the optical properties of QDs.

To consider the differences in the temperature dependence between QDs and nanoassemblies, one has to take into account that nanoassemblies at an initial molar ratio $x=\left[C_{\mathrm{CuP}}\right] /\left[C_{\mathrm{QD}}\right]=1$ were prepared by one-step mixing at ambient temperature. The thermodynamic formation of such self-aggregated assemblies is at high temperatures characterized by the corresponding complexation constant [39]. Estimations according to a Poisson distribution show that at $x=1$, the related probabilities $P_{i}$ of the number of dye 
molecules per QD are $P_{0}=0.3, P_{1}=0.4, P_{2}=0.3$, respectively. This implies, that some QDs are "porphyrin-free" without PL quenching at ambient temperature. Decrease of temperature leads probably to complete complexation of QDs because of the rise of the complexation constant at low temperatures [56]. Correspondingly, this should show up in more effective QD PL quenching. However, this is experimentally not reflected in an increase of PL intensity up to the phase transition. We cannot exclude that at the phase transition more nanoassemblies are formed, since dye molecules become "frozen" in the now rigid surfactant layer.

Summing up this part we conclude, that CdSe/ZnS QD undergo a phase transition in the sense, that the "freezing" ligand shell exerts strain on the ZnS shell thus creating trap states with reduced PL quantum yields. Moreover, this phase transition changes also the CdSe core absorption, which might be explained by a modification of the core structure. Notably, it has been shown recently [57] that the solvent environment and ligands not only help in confining the size of the QDs in solution but also stabilize the crystal structure of QDs by minimizing the surface free energy. In solution, the solvent molecules adsorbed on the QD surface can reduce the total surface free energy, and the surface can undergo reconstruction when the solvent molecules leave the QD surface. In turn, dye attachment creates new and/or more trap states, which obviously quench the PL very effectively. Phase transition temperature and the influence of dye induced trap states depend critically on the type of ligand and are more pronounced for TOPO as compared to amine ligands. This might be explained by the pyramidal structure of TOPO, which prohibits for steric reasons a complete passivation of the QD surface $[23,53]$. Now, that we have demonstrated, that dye molecules noticeably influence the optical properties at ligand controlled phase transitions, we will in the remaining part discuss the influence of attached dye molecules on the long-term photostability of CdSe/ZnS QDs.

\section{Photostability of QD-dye nanoassemblies}

The attachment of functionalized organic dyes to a QD surface manifests itself in noticeable QD PL quenching [13-21] as well as in related complex interface dynamics [26-28]. Obviously, PL quenching of the CdSe/ZnS constituent of the nanoassembly is a dynamic process caused by non-radiative relaxation channels for the exciton. We have quantitatively shown $[19,20,22,23,39]$ that the major part of the observed strong quenching of QD PL in nanoassemblies can neither be assigned to FRET nor to photoinduced charge transfer between the QD and the chromophore. This NonFRET quenching depends on QD size and ZnS shell thickness and is stronger for smaller quantum dots than for larger ones [22]. Based on the comparison of experimental data and quantum mechanical calculations it has been concluded that QD PL quenching in "QD-porphyrin" nanoassemblies can be understood in terms of the tunneling of the electron (of the excited electron-hole pair) across the ZnS shell followed by a (self-) localization of the electron or formation of trap states at the interface [22] or even in the matrix $[23,33]$. Our recent findings highlight that single functionalized molecules can be considered as extremely sensitive probes for the complex interface physics and dynamics of colloidal semiconductor QDs.

A modification of QD PL is also visible in single "QD-dye" experiments. Fig. 6 shows the comparison of blinking statistics for two samples spin coated from toluene solution onto quartz surface at $295 \mathrm{~K}$ : TOPO-capped CdSe/ZnS QDs and QD-(m-Pyr $)_{4} \mathrm{H}_{2} \mathrm{P}$ nanoassemblies both having the same initial $\mathrm{QD}$ concentration $\left(C_{\mathrm{QD}}=1.8 \times 10^{-9} \mathrm{M}\right.$, core diameter $d_{\mathrm{CdSe}}=3.2 \mathrm{~nm}, 3 \mathrm{ZnS}$ monolayers) and being excited within the QD first absorption band. Nanoassemblies were prepared at a molar ratio $x=\left[C_{\text {Porphyrin }}\right] /\left[C_{\mathrm{QD}}\right]=10$, at which the ensemble QD PL quenching is about $40 \%$ [19]. It is seen from Fig. $6 \mathrm{~A}$ and $\mathrm{B}$ that for both cases blinking statistics show
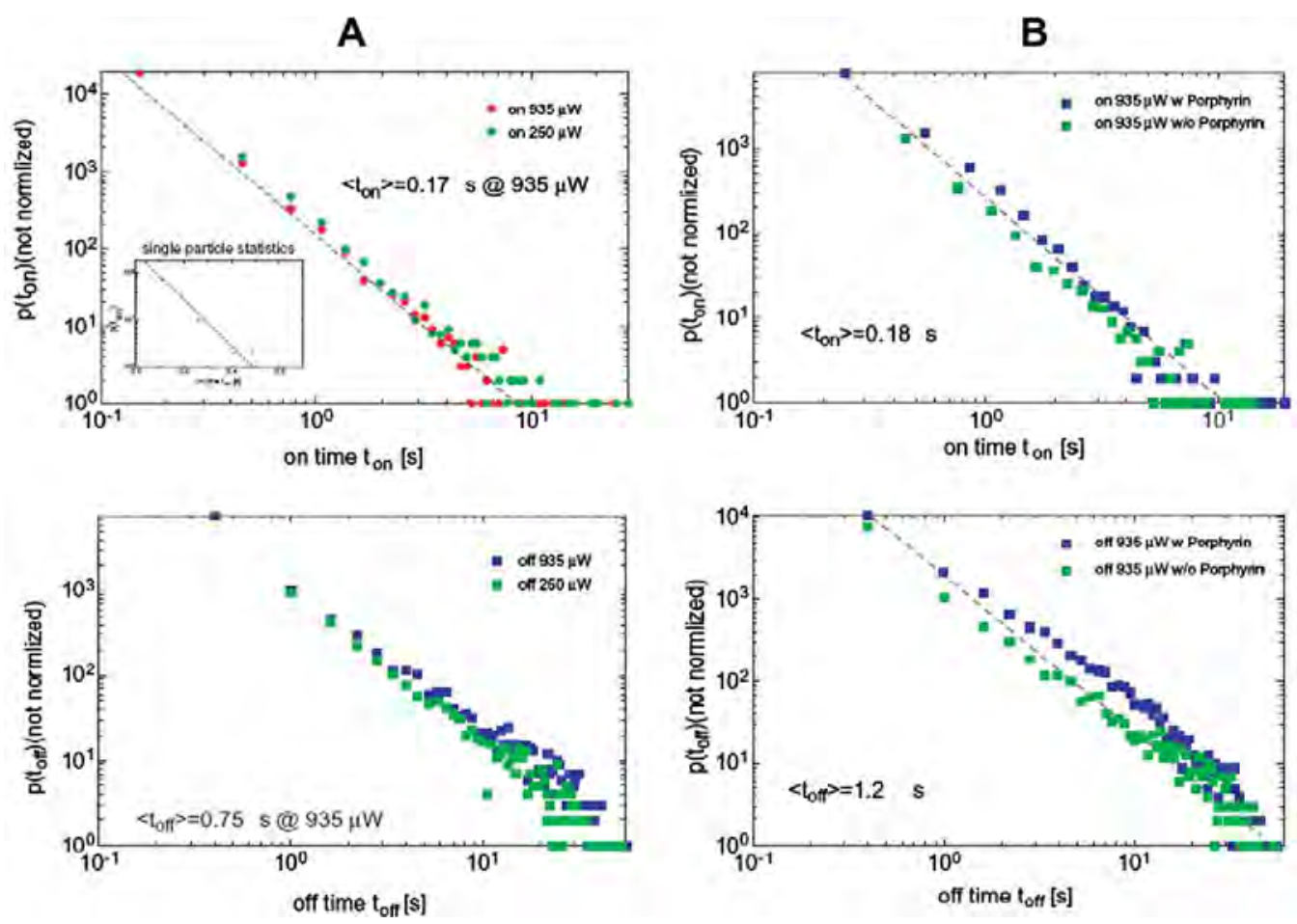

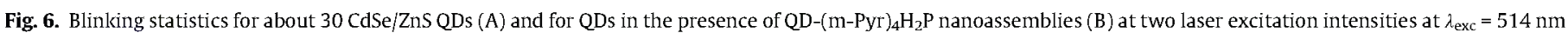
and $295 \mathrm{~K}$ [58]. The inset in the top left figure shows the statistic of one single QD. Average on- and off-times are indicated in each graph. 

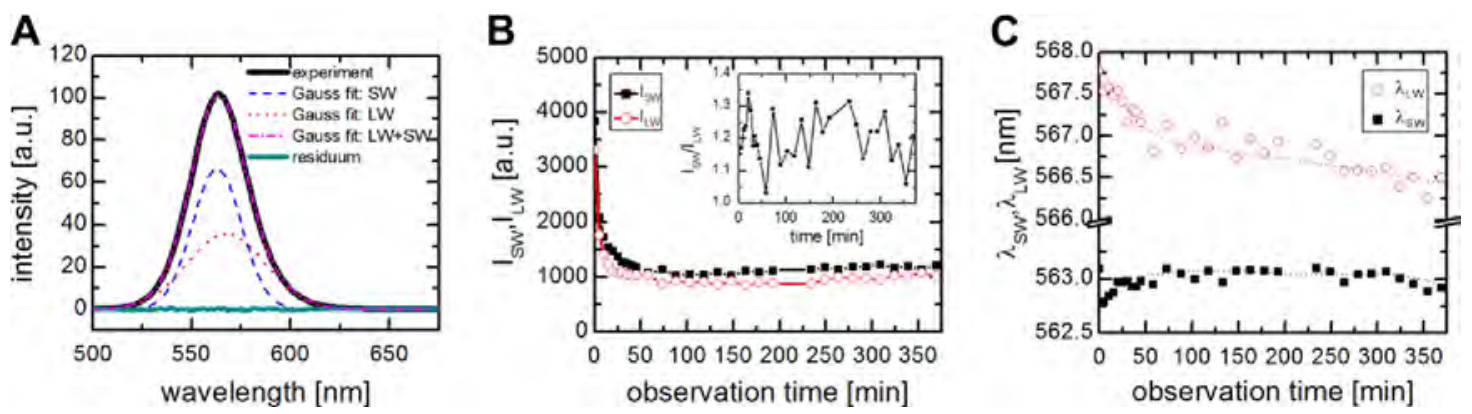

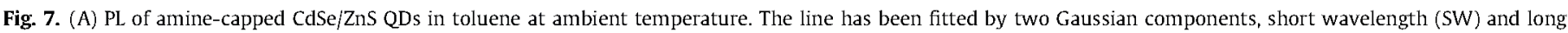

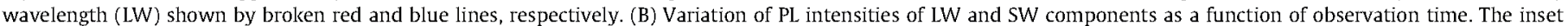
shows the related intensity ratio. (C) Variation of PL emission wavelength for SW and LW components as a function of observation time.
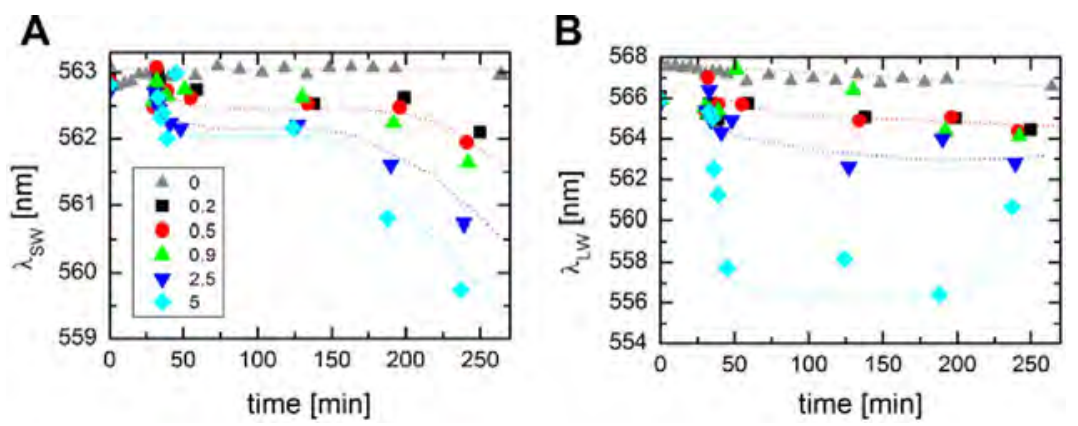

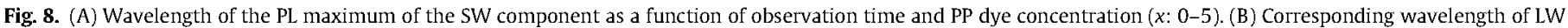
component. Lines are only guide lines.

a power law distribution for "on-" and "off-"times. Dark QD states are usually explained by charged nanocrystals [33], and the heterogeneity (power law behavior [59]) is inherent to broadly distributed (de-) population processes of the dark state. $\left\langle t_{\mathrm{on}}\right\rangle$ times of $0.18 \mathrm{~s}$ are the same in $\mathrm{QD}-(\mathrm{m}-\mathrm{Pyr})_{4} \mathrm{H}_{2} \mathrm{P}$ nanoassemblies and CdSe/ZnS QDs. In contrast, a substantial increase of $\left\langle t_{\text {off }}\right\rangle$ times is observed for nanoassemblies $\left(\left\langle t_{\mathrm{off}}\right\rangle=1.2 \mathrm{~s}\right.$ ) in comparison to $\left\langle t_{\text {off }}\right\rangle=0.75 \mathrm{~s}$ for QDs. These findings are considered as a proof of QD-porphyrin interactions leading to changed QD PL dynamics also on a single assembly level.

Before discussing the influence of perylene diimids on the PL for amine-capped CdSe/ZnS QDs, we first describe the bulk PL behavior for amine-capped CdSe/ZnS QDs. Fig. 7 shows the PL of an ensemble of amine capped CdSe/Zn QDs in toluene. For these QDs, the PL spectrum has to be fitted with two Gaussian components as is shown in Fig. 7A. We term the two components "short" (SW) and "long" (LW) wavelength lines. After sample preparation both the PL intensity and line shape/width change as a function of the waiting time. Fig. 7B shows for both spectral components PL temporal intensity changes as a function of the waiting time. The intensities decrease by about a factor 4 within 10 min and stay nearly constant there after. During the total time of several hours the integrated intensity ratio remains constant within experimental error (see inset Fig. 7B). However, the effective PL wavelengths of the 2 Gaussian lines change differently as can be seen in Fig. $7 \mathrm{C}$. While LW shifts systematically to the blue on a time scale of several hours, SW stays nearly constant. This can be interpreted as follows. We tentatively assign SW component to the near-bad edge PL, while LW component is assigned to a broad distribution of trap states. Obviously the width of the trap state distribution is reduced during the waiting time. Specifically, this implies that traps with the lowest PL energy are quenched more effectively. Noteworthy, TOPO-capped CdSe/ZnS QDs show similar spectral features. However, the detailed analysis of the properties of the related SW
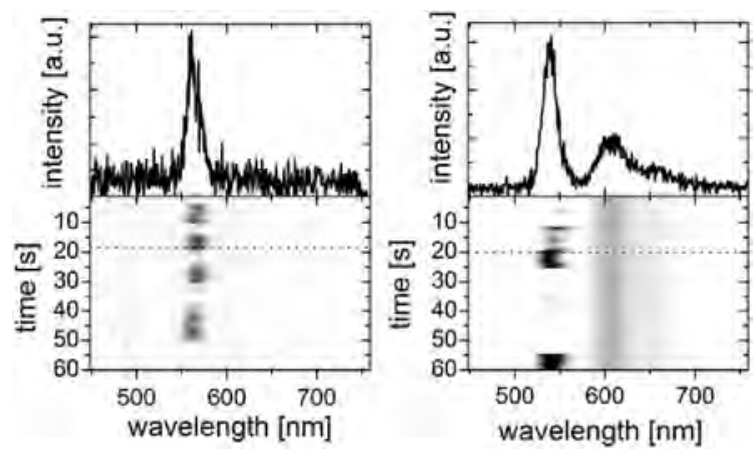

Fig. 9. Top: Single CdSe/ZnS PL emission spectrum (A) and luminescence spectrum of a single CdSe/ZnS and a single PP forming an assembly (B) at ambient temperature. Bottom: Luminescence intensities and wavelengths as a function of observation time. The broken lines show actually the frame (time) where the spectrum shown above is taken from. Time resolution is $1 \mathrm{~s}$. Single QDs and nanoassemblies QD + PP were excited with a repetition rate of $10 \mathrm{MHz}$ at $\lambda=465$ $\mathrm{nm}$ on the timescale of minutes with constant average excitation power of approximately $400 \mathrm{~W} / \mathrm{cm}^{2}$.

and LW components (decays, temperature 300-77 K dependence, etc.) for TOPO-capped CdSe/ZnS QDs is presently under investigation.

While the spectral shifts are extremely small $(1-2 \mathrm{~nm})$, they are enlarged following titration by (pyridyl) ${ }_{1}$-perylene diimide (PP) molecules. Fig. 8A and B shows the effect both for the $\lambda_{\text {sw }}$ and $\lambda_{L W}$ component. While the shift is at most $4 \mathrm{~nm}$ for $\lambda_{S W}$ it increases up to $12 \mathrm{~nm}$ for $\lambda_{L W}$. In both cases the shift becomes larger with increasing PP concentration. At molar ratio $x=5$ the experimental error becomes large since QD PL is already considerably quenched. The time scale of the respective shift becomes systematically shorter with increasing $x$. This can be understood in view of recent 

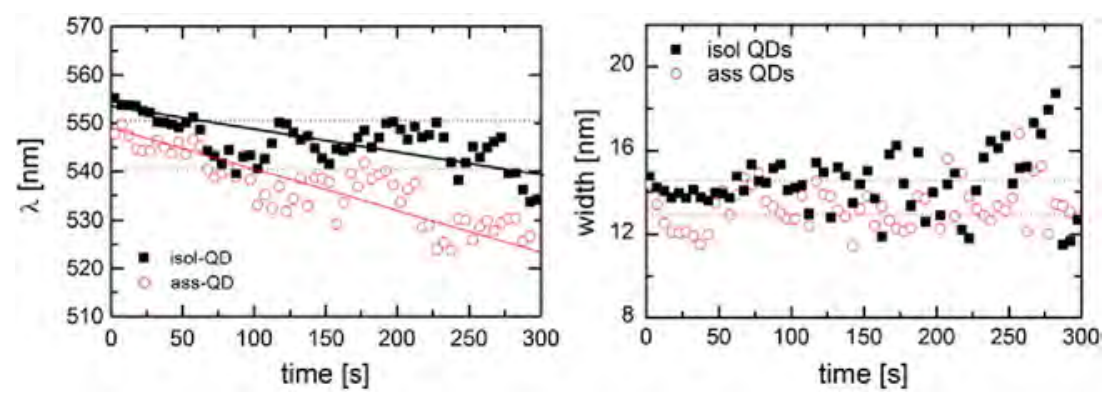

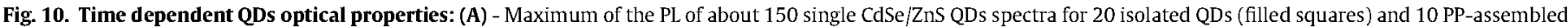
QDs (open circle). (B) - Corresponding PL line widths. The full lines are merely guide lines. The broken lines indicate the noise level.

findings of assembly formation as a function of concentration [23,32].

These observations indicate that assembly formation goes in line with increased bleaching of the long-wavelength component LW. We can, however, not exclude, that there is some cross-talk in the fitting procedure, which might lead to only an apparent blue shift of $\lambda_{S W}$. In that case all shifts have to be cast on $\lambda_{L W}$. Basically, also the titration experiments show, that the most red trap states are effectively bleached during observation time.

In the following we investigate the influence on the waiting time on the spectral properties of single QD-dye assemblies. For this purpose we have spincoated either amine-capped CdSe/ZnS or CdSe/ZnS + PP (at $x=1$ ) onto a glass substrate at ambient temperature. The PL spectrum of CdSe/ZnS (only QD PL spectrum in one focal laser spot) in Fig. 9A is shown in a time trace of the PL intensity during $60 \mathrm{~s}$ with $1 \mathrm{~s}$ resolution. We observe besides blinking a small blue shift. In Fig. 9B the (within one laser spot) superposition of QD and PP fluorescence shows a stable fluorescence of PP (at $\lambda=600 \mathrm{~nm}$ ), while the PL of the QD (at $\lambda=560 \mathrm{~nm}$ ) is blinking and blue shifting more effectively than in the absence of PP. We could select the spectra of both constituting components of the assembly only in a few cases within one laser spot in the confocal plane [32].

For sensitivity reasons it was only possible to fit the QD PL with only one Gaussian line contrary to the procedure reported for the ensemble. Fig. 10A shows the shift of the PL during waiting time for all single QD spectra. As can be seen on the time scale of the experiment the PL of nanoassembled CdSe/ZnS QDs shifts more effectively (by $25 \mathrm{~nm}$ ) to the blue than the one of alone CdSe/ZnS QDs $(\approx 17 \mathrm{~nm})$. Notably, the time-dependent blue shift is photoinduced, since isolated QDs were measured one after another and no systematic blue shift of the initial wavelength was observed for QDs recorded later after sample preparation. The line-width (see Fig. 10B) is becoming relatively narrower for nanoassemblies than for QDs though the experimental error is too large to draw confident conclusions.

The overall conclusion with respect to $\mathrm{CdSe} / \mathrm{ZnS}$ assemblies is that there is a noticeable difference in the photophysical behavior of QD PL upon assembly formation. Single particle detection indicates that even one attached PP molecule enhances the photodegradation considerably. However, we observe in parallel, that CdSe/ ZnS QDs are also though less photodegraded without PP.

Blue shifts of QD PL have been reported earlier [60] and have been assigned to photooxidation of the core material, which ends up in a smaller and thus more blue-emitting QD. However, our ensemble experiments are more convincingly interpreted in a way that predominantly one part of the PL (presumably related to deep trap states) is effectively quenched. Since this quenching is very effectively enhanced following nanoassembly formation, we assign the quenched trap states to surface related states, since they are expected to react most effectively with attached dye molecules. Single assembly experiments support this conclusion, but additionally show that also photooxidation of the CdSe core takes place since the PL is shifting to the blue by up to $20 \mathrm{~nm}$. This different behavior with respect to ensemble experiments might be related to the fact that during spin coating the surfactant layer is partly (or even completely) lost and the QD surface can be freely accessed by oxygen, which is often involved in photodegradation of QDs.

How can dye molecules enhance the photodegrading process? Two reasons can tentatively be envisaged. The first one is that upon attachment of dye molecules a large number of ligands are removed, which results in a more unprotected and thus more reactive surface, which allows for a stronger photodegradation either of surface states or even of the QD core. The second reason is related to the fact, that dye-induced trap states of QDs are more sensitive to photobleaching resulting in apparent spectral shifts and reduced integrated PL. This latter assumption is supported by our experiments on porphyrin-QD assemblies reported in the first part of this paper.

\section{Conclusions}

A combination of ensemble and single molecule spectroscopy of nanoassemblies formed from CdSe/ZnS QDs and two types of dye molecules revealed, that already very few or even only one attached dye molecule change the distribution and/or presence of dye related surface trap states considerably. This is manifested in the PL of the respective quantum dots. The "decoration" of QDs by dye molecules makes a phase transition of the QD ligand shell highly visible or even amplifies the phase transition. We conclude from absorption experiments, that the ligand phase transition has impact on the QD core structure and exciton-phonon coupling which will be investigated in more detail in forthcoming experiments. Finally we like to point out, that properties of QD-dye nanoassemblies are interesting in itself, but also provide a valuable tool to investigate surface related phenomena in QDs on an extremely low level of the surface modification.

\section{Acknowledgments}

We like to thank F. Wuerthner, Wuerzburg University for supplying us with perylene diimide dyes. Data related to single assembly blinking have been presented by $\mathrm{F}$. Cichos and $\mathrm{A}$. Issac, TU Chemnitz, on the occasion of the Spring Meeting of the German Physical Society in Regensburg, 2004. This work was funded by the Volkswagen Foundation (VW Grant I/79435 within the Priority Program "Physics, Chemistry and Biology with Single Molecules"), the Belorussian State Programs for Scientific Research "Convergence 3.2.08 - Photophysics of Bioconjugates, Semiconductor and Metallic Nanostructures and Supramolecular Complexes and Their Biomedical Applications" "Electronics and Photonics 2.3.03 - Photonics of Biological and Artificial Multimolecular Systems", as well 
as the Belorussian Foundation for Fundamental Research - Grant No. $\Phi 10 C 0-005$ “Development and Studying Physico-Chemical Properties of Composite Materials Based on Semiconductor Nanocrystals and Functional Organic Ligands".

\section{Appendix A. Supplementary material}

Supplementary data associated with this article can be found, in the online version, at doi:10.1016/j.chemphys.2012.02.008.

\section{References}

[1] C. de Mello, Chem. Soc. Rev. 40 (2011) 1512

[2] D.V. Talapin, J.-S. Lee, M.V. Kovalenko, E.V. Shevchenko, Chem. Rev. 110 (2010) 389

[3] D. Mocatta, G. Cohen, J. Schattner, O. Millo, E. Rabani, U. Banin, Science 332 (2011) 77.

[4] G.-X. Liang, L.-L. Li, H.-Y. Liu, J.-R. Zhang, C. Burda, J.-J. Zhu, Chem. Commun. 46 (2010) 2974.

[5] H.-M. Cheng, Chem. Commun. 47 (2011) 6763.

[6] U. Woggon, Optical Properties of Semiconductor Quantum Dots, Springer, Berlin, 2006.

[7] A.L. Rogach (Ed.), Semiconductor Nanocrystal Quantum Dots (Synthesis, Assembly, Spectroscopy and Applications), Springer-Verlag, Wien, 2008.

[8] H. Koo, M.S. Huh, J.H. Ryu, D.-E. Lee, I.-C. Sun, K. Choi, K. Kim, I.C. Kwon, Nanotoday 6 (2011) 204.

[9] D.F. Moyano, V.M. Rotello, Langmuir 27 (2011) 10376.

[10] L. Gur, N.A. Fromer, C.-P. Chen, A.G. Kanaras, A.P. Alivisatos, Nano Lett. 7 (2007) 409.

[11] M. McDowell, A.E. Wright, N.l. Hammer, Materials 3 (2010) 614

[12] L. Liu, J. Hensel, R.C. Fitzmorris, Y. Li, J.Z. Zhang, J. Phys. Chem. Lett. 1 (2010) 155

[13] C. Landes, C. Burda, M. Braun, M.A. El-Sayed, J. Phys. Chem. B 105 (2001) 2981.

[14] O. Schmelz, A. Mews, T. Basche, A. Herrmann, K. Muellen, Langmuir 17 (2001) 2861.

[15] A. Issac, S. Jin, T. Lian, J. Am. Chem. Soc. 130 (2008) 11280

[16] R. Clapp, I.L. Medintz, J.M. Mauro, B.R. Fisher, M.G. Bawendi, H. Mattoussi, J. Am. Chem. Soc. 126 (2004) 301.

[17] S. Dayal, C. Burda, J. Am. Chem. Soc. 129 (2007) 7977

[18] J. Lee, H.-J. Kim, T. Chen, K. Lee, K.-S. Kim, S.C. Glotzer, N.A. Kotov, J. Phys. Chem. 113 (2009) 109.

[19] E. Zenkevich, F. Cichos, A. Shulga, E. Petrov, T. Blaudeck, C. von Borczyskowski, J. Phys. Chem. B 109 (2005) 8679.

[20] E.l. Zenkevich, E.I. Sagun, A.A. Yarovoi, A.M. Shulga, V.N. Knyukshto, A.P. Stupak, C. von Borczyskowski, Opt. Spectrosc. 103 (2007) 998.

[21] Z-D. Qi, D-W. Li, P. Jiang, F-L. Jiang, Y-S. Li, Y. Liu, W-K. Wong, K.-W. Cheah, J. Mater. Chem. 21 (2011) 2455.

[22] T. Blaudeck, E. Zenkevich, F. Cichos, C. von Borczyskowski, J. Phys. Chem. C 112 (2008) 20251

[23] T. Blaudeck, E. Zenkevich, M. Abdel-Mottaleb, K. Szwaykowska, D. Kowerko, F. Cichos, C. von Borczyskowski, ChemPhysChem 12 (2011), doi:10.1002/ cphc.201100711.

[24] M. Anni, L. Manna, R. Cigolani, D. Valerini, A. Creti, M. Lomascolo, Appl. Phys. Lett. 85 (2004) 4169.
[25] I. Potapova, R. Mruk, C. Hubner, R. Zentel, T. Baschê, A. Mews, Angew. Chem. 117 (2005) 2490.

[26] T. Ren, P.K. Mandal, W. Erker, Z. Liu, Y. Avlasevich, L. Puhl, K. Mullen, T. Basche, J. Am. Chem. Soc. 130 (2008) 17242.

[27] G. Kaluzhny, R.W. Murray, J. Phys. Chem. B 109 (2005) 7012.

[28] A.M. Munro, D.S. Ginger, Nano Lett. 8 (2008) 2585.

[29] J. Frenzel, J.-O. Joswig, G. Seifert, J. Phys. Chem. C 111 (2007) 10761

[30] O. Voznyy, J. Phys. Chem. C 115 (2011) 15927.

[31] V. Klimov, in: H.S. Nalwa (Ed.), Handbook of Nanostructured Materials and Nanotechnology, vol. 4, Acad. Press, 2000, pp. 451-527.

[32] D. Kowerko, J. Schuster, N. Amecke, M. Abdel-Mottaleb, R. Dobrawa, F. Wuerthner, C. von Borczyskowski, Phys. Chem. Chem. Phys. 12 (2010) 4112

[33] A. Issac, C. von Borczyskowski, F. Cichos, Phys. Rev. B 71 (2005) 161302 (R).

[34] S.A. Crooker, T. Barrick, J.A. Hollinthworth, V.I. Klimov, Appl. Phys. Lett. 82 (2003) 2793.

[35] O. Labeau, P. Tamarat, B. Lounis, Phys. Rev. Lett. 90 (2003) 257404.

[36] C. De Mello Donega, M. Bode, A. Meijerink, Phys. Rev. B 74 (2006) 085320.

[37] M. Califano, A. Franceschetti, A. Zunger, Nano Lett. 5 (2005) 2360.

[38] E.I. Zenkevich, C. von Borczyskowski, in: S.K. Tripathy, J. Kumar, H.S. Nalwa (Eds.), Handbook of Polyelectrolytes and Their Applications, vol. 2, Amer. Sci. Publ., USA, 2002, pp. 301-348.

[39] E.I. Zenkevich, T. Blaudeck, A.M. Shulga, F. Cichos, C. von Borczyskowski, J. Luminesc. 122-123 (2007) 784

[40] R. Dobrawa, F. Wurthner, Chem. Commun. (2002) 1878

[41] E. Lang, F. Wurthner, J. Kohler, ChemPhysChem 6 (2005) 935.

[42] S. Murov, I. Carmichael, G. Hug, Handbook of Photochemistry, Marcel Dekker, New York, 1993.

[43] Y. Marcus, Solvent mixtures, Marcel Dekker, New York, 2002.

[44] E.P. Petrov, F. Cichos, C. von Borczyskowski, ]. Luminesc. 119-120 (2006) 412.

[45] D. Valerini, A. Creti, M. Lomascolo, L. Manna, R. Cingolani, M. Anni, Phys. Rev. B 71 (2005) 235409.

[46] T.J. Liptay, L.F. Marshall, P.S. Rao, R.J. Ram, M.G. Bawendi, Phys. Rev. B 76 (2007) 155314

[47] V.l. Klimov, Annu. Rev. Phys. Chem. 58 (2007) 635.

[48] T.J. Liptay, R.J. Ram, Appl. Phys. Lett. 89 (2006) 223132

[49] M.J. Fernee, B.N. Littleton, S. Cooper, H. Rubinsztein-Dunlop, D.E. Gomez, P. Mulvaney, J. Phys. Chem. C 112 (2008) 1878.

[50] G. Morello, M. De Giorgi, S. Kudera, L. Manna, R. Cingolani, M. Anni, J. Phys. Chem. C 111 (2007) 5846.

[51] S.F. Wuister, C. de Mello Donega, M. Bode, A. Meijerink, J. Am. Chem. Soc. 126 (2004) 10397.

[52] X. Peng, M.C. Schlamp, A.V. Kadavanich, A.P. Alivisatos, J. Am. Chem. Soc. 119 (1997) 7019

[53] B.o. Dabbousi, J. Redriguez-Vejo, F.V. Mikulec, J.R. Heine, H. Mattousi, R. Ober K.F. Jensen, M.G. Bawendi, J. Phys. Chem. B 101 (1997) 9463.

[54] Y.W. Cao, U. Banin, J. Am. Chem. Soc. 122 (2000) 9692.

[55] D. Kim, D. Holten, M. Gouterman, J. Am. Chem. Soc. 106 (1984) 2793.

[56] M. Meloun, J. Havel, E. Hogfeldt, Computation of Solution Equilibria, Ellis Horwood, Chichester, 1988

[57] A.S. Karakoti, S. Sanghavi, P. Nachimuthu, P. Yang, S. Thevuthasan, J. Phys. Chem. Lett. 2 (2011) 2925.

[58] A. Issac, F. Cichos. Deutsche Physikalische Gesellschaft (DPG), Spring Meeting of the Division of Condensed Matter Physics in Regensburg, 2004. Private Communication.

[59] F. Cichos, C. von Borczyskowski, M. Orrit, Curr. Opin. Colloid Interface Sci. 12 (2007) 272.

[60] W.G.J.H.M. van Sark, P.L.T.M. Frederix, A.A. Bol, H.C. Gerritsen, A. Meijerink, ChemPhyschem 3 (2002) 871. 